Caroline Kramer \& Linda Bauer

\title{
Das Standortnetz von Grundschulen in Baden-Württemberg im Wandel - Entwicklungen und Einflussfaktoren
}

\section{Zusammenfassung}

Das Standortnetz der Grundschulen in Baden-Württemberg hat in den vergangenen fünfzig Jahren Zentralisierungs- und Dezentralisierungsprozesse erlebt. Mit kleinräumigen Daten auf Gemeindeebene können zeit-räumliche Muster und Typen der Standortentwicklung aufgezeigt werden. Neben den demographischen Einflussfaktoren lassen sich bildungspolitische und pädagogische Leitbilder sowie ökonomisch motivierte Argumentationen als prägend für die Entwicklung des Schulstandortnetzes identifizieren.

Schlüsselwörter: Grundschulen, Kleinschulen, Standortnetz, Zentralisierung, Leitbilder, Privatisierung

\section{Baden-Württemberg's Changing Network of Primary School Locations - Developments and Influencing Factors}

\section{Abstract}

In the past fifty years Baden-Württemberg's network of primary school locations has experienced both centralization and decentralization processes. Based on local level data, time-spatial patterns and types of location developments can be uncovered. Factors influencing these processes are demographics, educational policy and pedagogical guiding principles as well as economically motivated arguments.

Keywords: primary schools, small schools, location networks, centralization, guiding principles, privatization 


\section{Einleitung}

Das Standortnetz der Grundschulen in Baden-Württemberg weist seit der Nachkriegszeit unterschiedliche Phasen von Zentralisierungs- und Dezentralisierungsprozessen auf. Mithilfe langer Zeitreihen der amtlichen Statistik, die mit einem hohen Detaillierungsgrad für jede Grundschule vorliegen, können diese Prozesse auf regionaler und lokaler Maßstabsebene sehr detailliert beschrieben werden. Somit lassen sich unterschiedliche zeitliche Phasen und besonders deren regional unterschiedliche Ausprägungen identifizieren. Darüber hinaus können mit Hilfe dieser Darstellungen räumliche Cluster erkannt werden, die Interpretationen und Erklärungen dieser Prozesse ermöglichen.

Im Folgenden wird in Kapitel 2 auf die spezifisch bildungsgeographischen Fragestellungen dieses Beitrags sowie die in diesem Beitrag verwendeten Daten der amtlichen Statistik eingegangen. Im dritten Kapitel werden anhand der baden-württembergischen Daten zu verschiedenen Zeitschnitten das Standortnetz von Grundschulen und die Entwicklung der Schülerzahlen ${ }^{1}$ dargestellt. Die bildungspolitischen Entscheidungen in den unterschiedlichen Phasen stehen in Kapitel vier im Fokus, wobei auch thematisiert wird, wie sie begründet wurden bzw. derzeit begründet werden. Im letzten - fünften - Kapitel werden die zentralen Ergebnisse zusammengefasst und Forschungsdesiderata formuliert.

\section{Bildungsgeographische Fragestellungen zum Standortnetz von Grundschulen}

Die spezifische Perspektive der Bildungsgeographie richtet sich auf bildungsbezogene Strukturen und Prozesse in ihren räumlichen und zeitlichen Ausprägungen. ${ }^{2}$ Dabei sind Fragen zur räumlichen Verteilung von Bildungseinrichtungen, zu ihrem Zugang und $\mathrm{zu}$ ihrer Bedeutung für ihre räumliche Umgebung schon seit Beginn der Bildungsgeographie (Meusburger, 1998) bedeutsam. Dem Leitbild der „gleichwertigen Lebensverhältnisse“ folgend, das in $\$ 1$, Abs. 2 des Raumordnungsgesetzes (Bundesministerium für Justiz und Verbraucherschutz \& Bundesamt für Justiz, 2008) festgeschrieben ist, zählen eine flächendeckende Bildungsversorgung und eine gute Erreichbarkeit von Bildungseinrichtungen zu den zentralen Infrastrukturen, die die öffentliche Hand den Bürger*innen zur Verfügung stellen soll. Über diesen klassischen Ansatz der Daseinsvorsorge hinaus sind jedoch auch Konzepte der räumlichen Verankerung (Werlen, 2000) und der raumbezogenen Identität (Weichhart, Weiske \& Werlen, 2006) für bildungsgeographische Untersuchungen von Schulstandortnetzen

1 Wenn von "Schülerzahlen“ gesprochen wird, ist immer die Zahl der Schülerinnen und Schüler gemeint.

2 Siehe dazu Freytag, Jahnke \& Kramer, 2015, und Freytag, Jahnke \& Kramer, 2016, S. 140 ff. 
und deren Planung bedeutsam. Diese Konzepte kommen dann zum Einsatz, wenn z. B. die Rolle der Schule und der Lehrkräfte für das soziale und kulturelle Leben von Gemeinden thematisiert wird. Da bildungspolitische Konzepte und Planungen auf der Landesebene in Gemeinden und Ortsteilen ihre Wirkung entfalten, ist ein wesentliches Kennzeichen bildungsgeographischer Studien, sowohl die verschiedenen räumlichen Ebenen als auch die dort anzutreffenden Akteure in den Blick zu nehmen.

Vor diesem Hintergrund wird der bildungsgeographische Blick, der in diesem Beitrag auf das Standortnetz von Grundschulen eingenommen wird, von folgenden Fragestellungen geleitet:

- Wie hat sich das Standortnetz der Grundschulen in den vergangenen fünf Jahrzehnten entwickelt? Welche organisatorischen Veränderungen im Bildungssystem haben Einfluss auf dieses Schulstandortnetz?

- Wie hat sich die Zahl und Verteilung der Schüler*innen in diesem Zeitraum entwickelt? Welche Einflussfaktoren auf das Schulstandortnetz sind auf welchen räumlichen Ebenen zu erkennen?

- Welche Auswertungsmöglichkeiten bietet die amtliche Statistik, und wie können diese die Bildungsplanung unterstützen?

- Wie gestaltet die baden-württembergische Bildungspolitik und -planung das Standortnetz der Grundschulen?

Die Frage nach der Entwicklung der Standortnetze ist nicht nur unter dem Aspekt der Daseinsvorsorge und der Infrastrukturausstattung zu stellen, sondern es sind damit auch Fragen der Bildungsgerechtigkeit verbunden. Mit der Distanz zu Schulen und ihrer Erreichbarkeit sind häufig auch individuelle Bildungschancen und damit potenzielle Ungleichheiten verknüpft, die nicht nur Kinder aus bildungsfernen Haushalten treffen (Fickermann, 1997, S. 149; Fickermann, Schulzeck \& Weishaupt, 2002, S. 42; Sixt, 2010, S. 218). Auch wenn auf diesen Aspekt in dem vorliegenden Beitrag nicht ausführlich eingegangen werden kann, so stellt er einen weiteren wichtigen Grund dar, die Standortnetze von Bildungseinrichtungen genauer zu betrachten.

Analysiert man die Veränderungen eines Standortnetzes von Schulen, so können grundsätzlich zwei gegenläufige räumliche Prozesse unterschieden werden, nämlich Zentralisierungs- und Dezentralisierungsprozesse (vgl. Abb. 1). Im ersten Fall werden Schulen - in der Regel an peripheren Standorten - geschlossen und gleichzeitig Institutionen an zentralen Standorten vergrößert. Zugleich wird ihr Einzugsbereich vergrößert, und die Schulwege werden länger. Im zweiten Fall finden im Zuge von Dezentralisierungsprozessen Neugründungen oder Wiedereröffnungen von Schulen in Orten statt, an denen zuvor keine Schule (mehr) war. Damit gehen in der Regel kürzere Schulwege und kleinere Einzugsgebiete einher. Meist finden Zentralisierungsprozesse bei sinkenden Schülerzahlen statt, und umgekehrt kommt es häufiger zu Dezentralisierungsprozessen bei steigenden Schülerzahlen (vgl. fett gerahmte Felder in Abb. 1). 
Abb. 1: Typen der Standortentwicklung

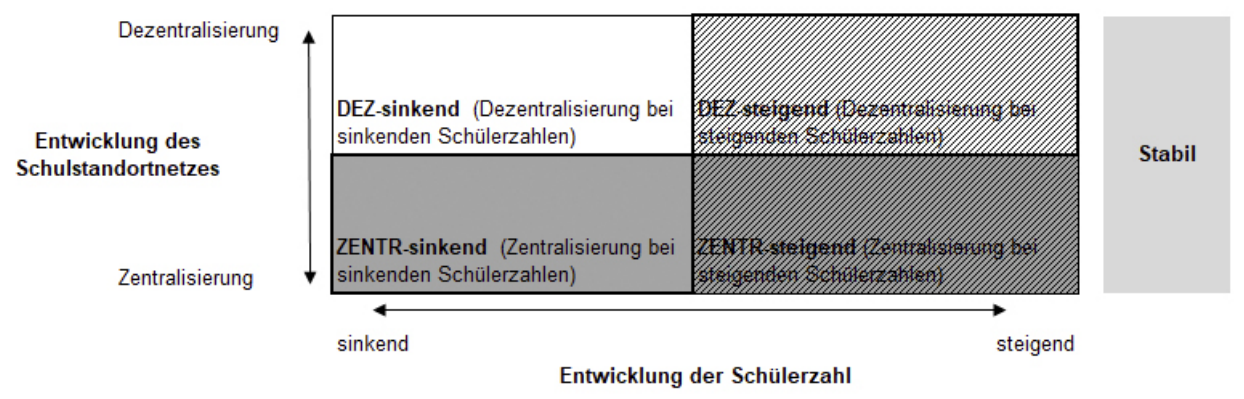

Quelle: eigener Entwurf nach Bauer, 2016, S. 89

Da im Rahmen von Zentralisierungsprozessen grundsätzlich zuerst kleine Schulen geschlossen werden, gilt ihnen in diesem Beitrag besondere Aufmerksamkeit. Ihr Erhalt ist zum einen davon abhängig, dass eine ausreichende Zahl schulpflichtiger Kinder in ihrem Einzugsgebiet lebt. Aus diesem Grund besteht die Gefahr, dass diese Schulen bei rückläufigen Geburtenzahlen und Abwanderungen junger Menschen die Mindestgröße nicht mehr erreichen und dann in der Regel eine Schließung der Schule vorgeschlagen wird. Dies ist seit der Wiedervereinigung in den neuen Bundesländern zu beobachten (Fickermann, Schulzeck \& Weishaupt, 2002, S. 28 f.; Kann, 2016) und wird derzeit in Baden-Württemberg wieder diskutiert (vgl. Kap. 4). Zum anderen ist die Entscheidung über die Mindestgröße, die Mindestzahl der Parallelklassen (Mindestzügigkeit) und die Organisationsform (jahrgangsübergreifende oder nur nach Jahrgängen getrennte Klassen) eine ausschließlich bildungspolitische und -organisatorische Entscheidung. Daher gibt es weitaus mehr Spielräume für die Gestaltung des Schulstandortnetzes, als nur der Entwicklung der Schüler*innenzahl zu folgen (vgl. dazu auch Kann, 2016, S. 254).

Insbesondere der jahrgangsübergreifende Unterricht, bei dem in der Regel zwei Schülerjahrgänge in einer Klasse gemeinsam unterrichtet werden, stellt eine organisatorische Besonderheit dar, die seit den Reformschulbewegungen zu Beginn des 20. Jahrhunderts immer wieder zu kontroversen Diskussionen geführt hat (Fickermann, Weishaupt \& Zedler, 1998, S. 9 ff.) und u.a. unter der Bezeichnung „Landschuldiskussion“ (Diederich, 1967, S. 129) bekannt geworden ist. Die schulischen Leitbilder lassen sich im Wesentlichen zwei konträren Positionen zuordnen: a) die Verfechter*innen der kleinen, niedrig organisierten Schulen vor Ort (= Dezentralisierung) und b) die Verfechter*innen der großen Schulen mit Jahrgangsklassen am größeren und zentraleren Ort (= Zentralisierung). ${ }^{3}$ Während die Verfechter ${ }^{\star}$ innen der kleinen Schule die Vorteile des ganzheitlichen und fächerübergreifenden, lebens-

3 Diese Diskussion zu Vor- und Nachteilen kleiner Grundschulen wurde (Kramer, 1993; Fickermann, Weishaupt \& Zedler, 1998) und wird in vielen europäischen Ländern aufgrund des demographischen Wandels immer noch bzw. wieder intensiv geführt (Kvalsund \& Har- 
weltlich ausgerichteten Unterrichts, des sozialen Lernens und Helfens sowie die Nähe der Schule am Ort betonen, stellen die Verfechter*innen der großen, voll organisierten Schule den fachspezifischen Unterricht, größere Wahlmöglichkeiten und eine effizientere Gestaltung des Schulalltags in den Vordergrund. Die Vertreter*innen dieser beiden Leitbilder treffen in der Bildungspolitik oft auf unterschiedlichen räumlichen Ebenen aufeinander. Während die Verfechter*innen der kleinen Schulen meist im lokalen und regionalen Kontext (oder in den Bildungswissenschaften) anzutreffen sind, so sind auf Bezirks- und Landesebene deutlich häufiger Verfechter*innen der großen Schulen zu finden (vgl. Kap. 4).

Da Bildungsaufgaben im föderalen System der Bundesrepublik Deutschland zu den Hoheiten der Bundesländer zählen, werden auch die dazugehörigen Daten in den Statistischen Landesämtern gesammelt und verwaltet (https://www.statistik-bw.de). Für einen detaillierten Vergleich der Schulen zwischen 1991/92 und 2014/15 erhielten die Autorinnen eine Sonderauswertung des Statistischen Landesamtes BadenWürttemberg zu Schülerzahlen, Klassenzahlen und Standorten der einzelnen Schulen, so dass Auswertungen auf Gemeindeebene möglich waren. Dies hatte den Vorteil, dass Schulen, die organisatorisch nur als Außenstellen geführt werden und in den offiziellen Schulstatistiken wegfallen, jedoch häufig die einzige Schule an ihrem Standort sind, berücksichtigt werden konnten. Allerdings werden Schülerinnen unter dem Schulort geführt, so dass keine Aussage über den tatsächlichen Wohnort getroffen werden kann. Allerdings ist zu vermuten, dass die Abweichungen zwischen Schul- und Wohnort gering sind, da sich die Grundschulwahl weitgehend an vorgeschriebenen Schulbezirken orientiert und Eltern in Baden-Württemberg nur in Ausnahmefällen von dieser Vorgabe abweichen dürfen. Ein besonderes Augenmerk liegt auf den Schulen im ländlichen Raum. Die Definition des ländlichen Raums orientiert sich hierbei an den Raumkategorien auf Grundlage des Landesentwicklungsplanes 2002 für Baden-Württemberg, in dem Verdichtungsräume, Randzonen der Verdichtungsräume und ländlicher Raum unterschieden werden. Der ländliche Raum wird vorwiegend aufgrund der geringeren Siedlungsdichte von den beiden anderen Kategorien abgegrenzt (Wirtschaftsministerium Baden-Württemberg, 2002, S. 15). ${ }^{4}$ Zum ländlichen Raum in dieser Studie werden alle Kreise gezählt, die ganz oder mehrheitlich dem ländlichen Raum (im o.g. Sinne) zugehörig sind. Der ländliche Raum umfasst somit 18 Landkreise in Baden-Württemberg, welche sich überwiegend in den südlichen Regionen im Hochschwarzwald, in der Region BaarHeuberg, auf der Schwäbischen Alb und in der nordöstlichen Region HohenloheBauland befinden.

greaves, 2009; Åberg-Bengtsson, 2009; Raggl, 2015; Autti \& Hyry-Beihammer, 2014; Jahnke, Kramer \& Meusburger, 2019 (im Druck)).

4 Innerhalb des ländlichen Raums wird zwischen den Verdichtungsbereichen im ländlichen Raum und dem ländlichen Raum im engeren Sinne unterschieden. Dies geschieht anhand von Siedlungsverdichtung, Verflechtungen und Freiraumanteil (Wirtschaftsministerium Baden-Württemberg, 2002, S. 15). 


\section{Entwicklung des Schulstandortnetzes der Volks- und Grundschulen in Baden-Württemberg}

Das Standortnetz der baden-württembergischen Schulen hat in den vergangenen fünfzig Jahren mehrere Wechsel von Zentralisierungs- und Dezentralisierungsprozessen erfahren. Nach dem zweiten Weltkrieg war das Schulwesen in Baden-Württemberg - wie in vielen anderen Flächenstaaten der Bundesrepublik auch - noch von einer großen Zahl ein- und zweiklassiger Volksschulen geprägt. So wurden 1960 von den insgesamt 4.060 Volksschulen in Baden-Württemberg noch 52 Prozent der Schulen ein- und zweiklassig geführt (Kramer, 1993, S. $104 \mathrm{ff}$.). Die Volksschulen waren zu diesem Zeitpunkt nahezu flächendeckend über das Land verteilt, was daran zu erkennen ist, dass von den damals noch 3.381 Gemeinden des Landes Baden-Württemberg 97 Prozent über mindestens eine Volksschule verfügten (ebd., S. 105). ${ }^{5}$

Ab Mitte der 1960er-Jahre begann auch in Baden-Württemberg unter dem damaligen Kultusminister Hahn die erste große Bildungsreform, die durch starke Zentralisierungsprozesse und die Auflösung einer großen Zahl kleiner Schulen gekennzeichnet war (1965: 4.079 Schulen; 1974: 2.043 Schulen). Der Anteil der einklassigen Schulen sank in dieser Zeit von 41 Prozent auf 16 Prozent. Gleichzeitig erfolgte eine Gemeindereform, in der die Zahl der Gemeinden von 3.381 auf 1.111 Gemeinden reduziert wurde, von denen nach beiden Reformen 16 Prozent keine eigene Schule mehr besaßen. Diese parallel verlaufenden Zentralisierungsprozesse wurden von der Landespolitik als Modernisierungsprozess verstanden, der nicht zuletzt eine größere ökonomische Effizienz der Verwaltung zum Ziel hatte. Besonders deutlich wirkten sich diese Zentralisierungsprozesse in den ländlichen und peripheren Regionen Baden-Württembergs aus.

Ein Blick auf die unterschiedliche Entwicklung der Anzahl der Schulen und der Anzahl der Schüler*innen zeigt, dass das Jahrzehnt der Zentralisierung des Standortnetzes von Volksschulen von 1965 bis 1975 nicht mit einem Rückgang der Schülerzahlen einherging (Zentralisierung bei steigenden Schülerzahlen=ZENTRsteigend; vgl. Abb. 1, 2 und 5). Der sog. „Sputnik-Schock“ (1957) und die Studie von Picht (1964) zur „Bildungskatastrophe in Deutschland“ waren Auslöser für die Forderung nach Schulen, die die Anforderungen einer meritokratischen Gesellschaft erfüllen konnten. Als Vorbild galten die städtischen großen, nach Jahrgängen gegliederten Schulen; kleine Schulen mit jahrgangsübergreifendem Unterricht galten als rückständig. Diese Haltung war in dieser Zeit weit verbreitet und führte auch in zahlreichen anderen europäischen Ländern zu starken Konzentrationsprozessen im Schulstandortnetz (vgl. Fußnote 3). Da nach 1974 die Schülerzahlen sanken und der

$5 \mathrm{Zu}$ diesem Zeitpunkt wurden noch die Klassen 1-8 in einer Volksschule geführt und auch in den Statistiken als eine Schulform abgebildet. Mit dem Hamburger Abkommen wurde 1964 das allgemeinbildende Schulwesen in Deutschland vereinheitlicht. Erst ab diesem Zeitpunkt werden Grundschulen bis zur vierten Klasse als eigenständige Schulform geführt. 
Abb. 2: Zahl der Schüler*innen in Klasse 1 bis 4 an öffentlichen (Grund-)Schulen in BadenWürttemberg von 1959/60 bis 2016/17 und Entwicklung des Standortnetzes

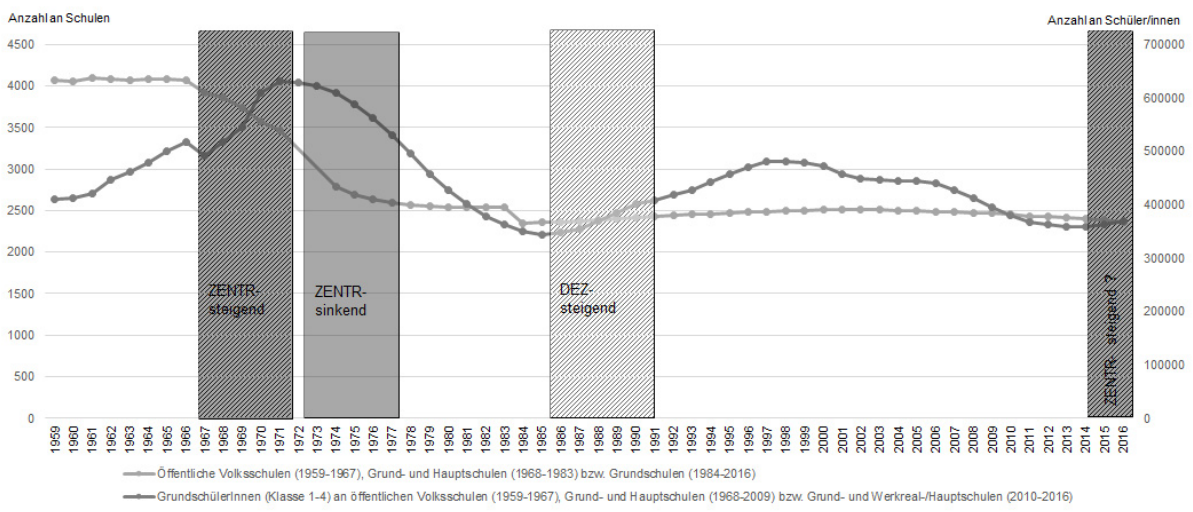

Anm.: ZENTR=Zentralisierung; DEZ=Dezentralisierung; steigend=steigende Schülerzahlen; sinkend= sinkende Schülerzahlen.

* Ab 1984 sind nur die Grundschulstandorte dargestellt.

Quelle: Statistisches Landesamt Baden-Württemberg (https://www.statistik-bw.de), Statistisches Bundesamt (https://www.destatis.de); eigene Berechnungen

Zentralisierungsprozess weiter voranschritt, kann diese Phase als Zentralisierung bei sinkenden Schülerzahlen (ZENTR-sinkend) bezeichnet werden.

Nach einem Jahrzehnt der Konsolidierung folgte im Jahr 1987 auf Initiative des baden-württembergischen Kultusministers Mayer-Vorfelder eine kurze Phase der Dezentralisierung des Standortnetzes der Grundschulen. Im Rahmen des Programms „Wiedereinrichtung wohnortnaher Grundschulen“ wurden bis 1991/92 insgesamt 119 kleine Grundschulen (wieder) eingerichtet. Dabei wurde betont, dass diese Schulen nicht mit den „Zwergschulen“ alter Prägung vergleichbar sein sollten. Während die Eltern diese Initiative auf Grund der kurzen Schulwege meist positiv bewerteten, standen die Kommunen ihr häufig skeptisch gegenüber, da sie die Kosten für die bauliche Wiedereinrichtung und den Erhalt der Schulen tragen mussten. Oft wurden in den Ortsteilen, in denen die Schule wieder eröffnet wurde, die Gebäude bereits anderweitig genutzt (z.B. für Vereine, die Feuerwehr), so dass diese Initiative nicht unerhebliche Kosten für die Gemeinden zur Folge hatte. Zu dieser Zeit war ein leichter Anstieg der Schülerzahlen abzusehen. Das Schulstandortnetz erfuhr einen Dezentralisierungsprozess bei steigenden Schülerzahlen (DEZ-steigend).

Grundschulen sind in Baden-Württemberg aktuell vielfältig organisiert. Sie können eigenständig oder im Verbund mit einer weiterführenden Schule - meist Werkrealschulen und Gemeinschaftsschulen - geführt werden. Zudem können Grundschulen in Baden-Württemberg als Außenstelle einer Stammschule organisiert sein. Die Herabstufung einer eigenständigen Schule zur Außenstelle erfolgt meist aus ökonomi- 
schen Gründen, da keine Schulleitung finanziert werden muss und Lehrkräfte mit der Stammschule geteilt werden können. Sie stellt oft den ersten Schritt zur späteren Schließung dar. Immer stärkeren Zuspruch finden in den letzten Jahren die Konzepte der verlässlichen Grundschule und der Ganztagesgrundschule, welche für die Attraktivität einer Grundschule entscheidend sein können. Verlässliche Grundschulen garantieren eine Betreuung der Kinder während der Stundenplanzeiten. In der Ganztagesgrundschule werden Schüler*innen an fest vereinbarten Wochentagen ganztägig betreut. Den Schülerinnen wird ein Mittagessen angeboten, und es werden Freizeitangebote, beispielsweise durch Sportvereine oder Musikschulen, geschaffen. Ziel dieser Konzepte ist es, die Vereinbarkeit von Familie und Beruf zu verbessern. Zudem soll damit für mehr Bildungsgerechtigkeit und bessere Lernleistungen bei den Schüler*innen gesorgt werden.

Für die Sicherung der Grundschulen in Baden-Württemberg ist ihre organisatorische Einbindung ein wesentlicher Standortfaktor. Nach den Analysen von Bauer (2016) wurden rund zwei Drittel der geschlossenen Schulen zuletzt als Außenstellen (ebd., S. 63) und zu 91 Prozent nicht im Verbund mit einer weiterführenden Schule geführt (ebd., S. 64). Außerdem ist es in den letzten Jahren zunehmend schwieriger geworden, Schulleiter*innen für Grundschulen zu finden, da die Besoldung nur unwesentlich höher ist als für Lehrkräfte, damit nur eine geringe Deputatsermäßigung verbunden ist, dafür aber wesentlich mehr Verwaltungsarbeit anfällt. Aus diesen Gründen waren im April 2018 in Baden-Württemberg 251 Schulleitungsstellen vakant, davon allein 164 Stellen an Grundschulen, die meist in ländlichen Regionen liegen (vgl. Lehrer Online in Baden-Württemberg). Es muss an dieser Stelle erwähnt werden, dass Personalmangel in allen Zentralisierungsphasen als Argument für eine Schulschließung genutzt wurde, er jedoch oft auf unattraktive Arbeitsbedingungen zurückzuführen ist.

Um die Entwicklung des Schulstandortnetzes präzise zu analysieren, ist es notwendig, die Zahl der Schulen an ihren jeweiligen Standorten zu ermitteln. Aufgrund der zahlreichen Schulreformen in den vergangenen Jahren in Baden-Württemberg (Auflösung der Hauptschule, Einführung der Werkrealschule (und Wiederauflösung) sowie der Gemeinschaftsschule) ist es nicht leicht, die Zahl der Grundschulstandorte verlässlich zu ermitteln, da diese im ersten Zeitschnitt noch mehrheitlich in Kombination mit weiterführenden Schulen geführt wurden. Bauer (2016) hat diese Daten für den Vergleich der Grundschulstandorte zwischen den Schuljahren 1991/92 und 2014/15 aufbereitet; dieser ist Grundlage der nachfolgenden Analysen.

Auch wenn die Zahl der Grundschulen für das gesamte Bundesland in diesem Zeitraum kaum Veränderungen erfährt (vgl. Abb. 2) und trotz insgesamt sinkender Schülerzahlen im Ganzen stabil bleibt, sind dennoch große regionale Unterschiede festzustellen (vgl. Abb. 3). Die Standortnetze in den ländlichen Regionen im Süden des Landes erfahren im Beobachtungszeitraum eine deutliche Ausdünnung, wo- 
von besonders Kleinstädte und Landgemeinden betroffen sind (73 Prozent aller Schließungen betreffen Gemeinden dieser Kategorie). Nur die Kreise im Hochschwarzwald, wo sehr periphere Lagen und winterliche Schulwegbedingungen prägend sind, bleiben von Schließungen verschont. Insgesamt werden in diesem Zeitraum 147 Grundschulstandorte geschlossen, wobei der periphere Südosten dabei stärker betroffen ist als der periphere Nordosten. ${ }^{6}$

Abb. 3: Grundschulschließungen in Baden-Württemberg zwischen 1991/92 und 2014/15

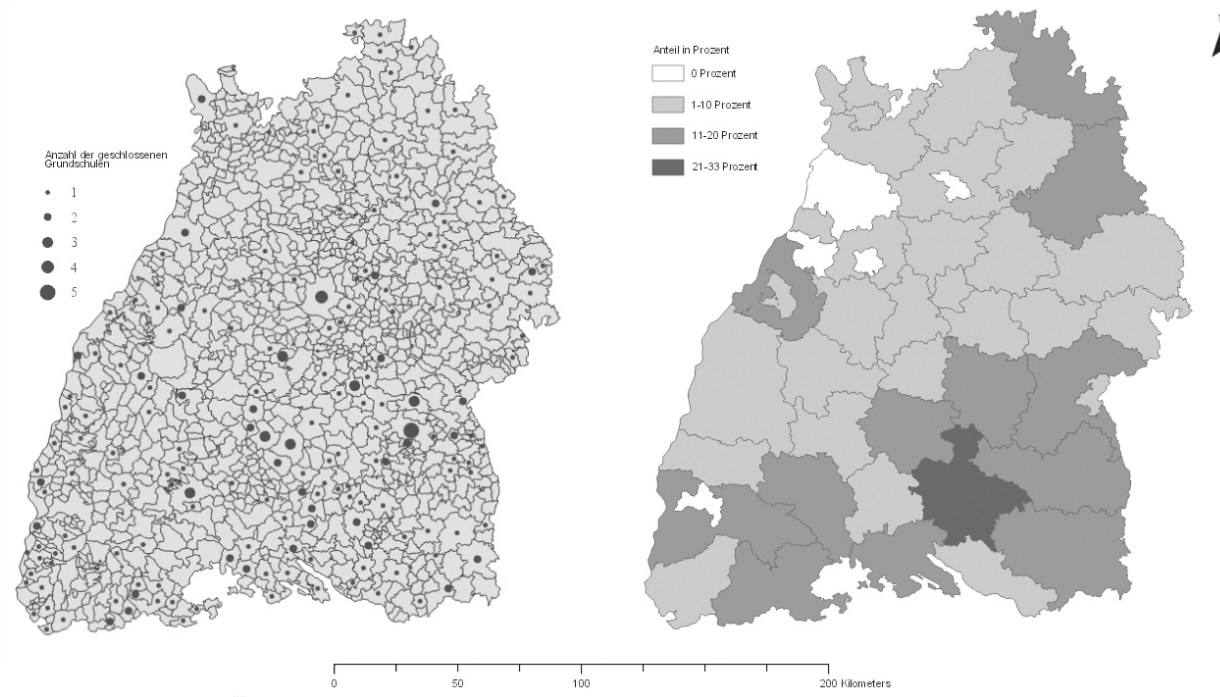

Anm.: Linke Karte: Anzahl der öffentlichen Grundschulen, die zwischen 1991/92 und 2014/15 geschlossen wurden; rechte Karte: Prozentualer Anteil der Anzahl der öffentlichen Grundschulen, die zwischen 1991/92 und 2014/15 geschlossen wurden, an allen öffentlichen Grundschulen des Kreises.

Quelle: eigener Entwurf nach Bauer, 2016, S. 61

Eine Zunahme an Grundschulen erfahren fast ausschließlich die Groß- und Mittelstädte in Baden-Württemberg, allen voran Stuttgart (14 zusätzliche Schulen, davon 12 privat), Freiburg (13, davon 10 privat), Heidelberg (8, davon 7 privat) und Karlsruhe (6, davon 5 privat). Während von den geschlossenen Schulen von 1991/92 bis 2014/15 nur eine Schule privat geführt wurde, waren von neu eröffneten Grundschulen 57 Prozent (= 119 Schulen) in öffentlicher und 43 Prozent (= 90 Schulen) in privater Hand. In den Stadtkreisen Freiburg und Heidelberg machen private Grundschulen bereits rund ein Viertel aller Grundschulen aus. Darauf wird in Kapitel 4 näher eingegangen.

6 Da hier Außenstellen mitgezählt wurden, ist die Zahl der Schulen deutlich höher als in Abb. 2. 
Neben den Grundschulschließungen und -eröffnungen sind die im Untersuchungszeitraum stattgefundenen Umstrukturierungen durch Schulreformen bemerkenswert, da sie latent auch Auswirkungen auf das Standortnetz der Grundschulen haben. Während 1991/92 noch die Mehrheit der Grundschulen mit einer Schule im Sekundarschulbereich zusammengeführt wurde, so trifft dies 2014/15 nur noch auf rund ein Viertel der Schulen zu. Der Konzentrationsprozess im Schulwesen betrifft somit in dieser Phase vorwiegend nur die weiterführenden Schulen. Der Erhalt der Grundschulen in kleinen Gemeinden ist aus vielerlei Sicht umso mehr langfristig für die Entwicklung des ländlichen Raums von großer Bedeutung.

Abb. 4: Vergleich der prozentualen Veränderung der Anzahl der Grundschulstandorte und Anzahl der Grundschüler*innen (öffentliche Grundschulen)

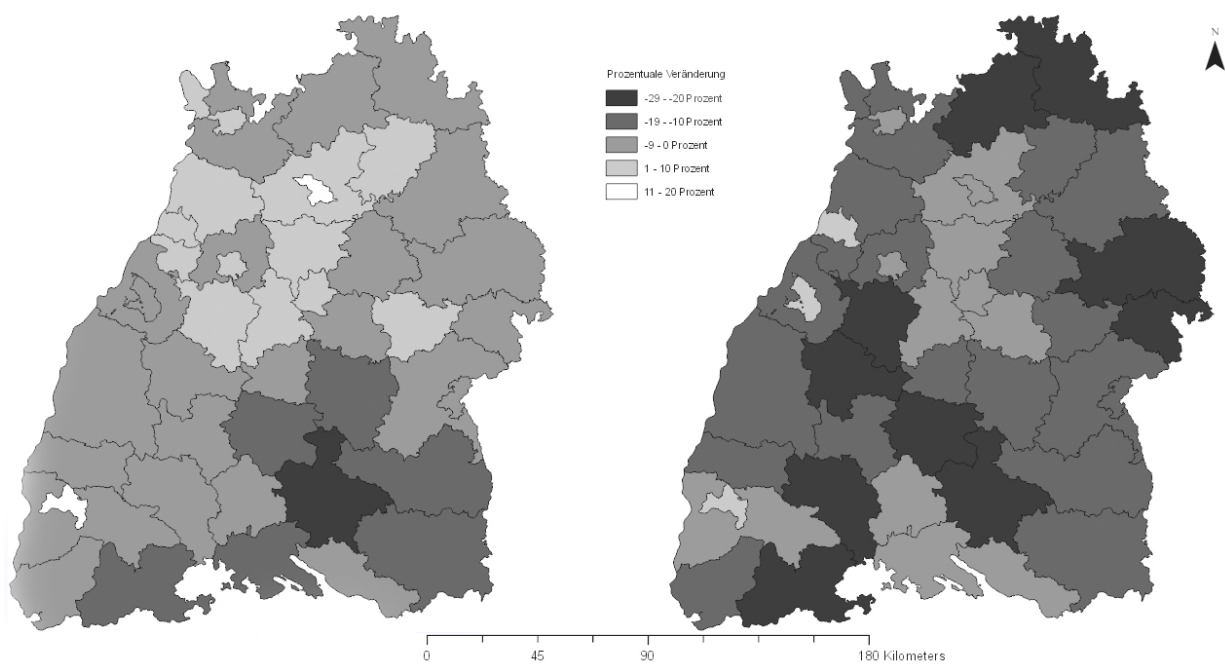

Anm.: Linke Karte: Prozentuale Veränderung der Anzahl der öffentlichen Grundschulen zwischen 1991/92 und 2014/15; rechte Karte: Prozentuale Veränderung der Anzahl der Grundschüler*innen an öffentlichen Grundschulen zwischen 1991/92 und 2014/15.

Quelle: eigener Entwurf nach Bauer, 2016, S. 81

Vergleicht man die regional differenzierte Entwicklung der Zahl der Schulen mit der Schülerzahl (Abb. 4), so wird deutlich, dass nur für zehn Kreise die Klassen für beide Karten in der prozentualen Entwicklung übereinstimmen. Vor allem in den ländlich geprägten Kreisen im Nordosten und im Süden des Landes geht die Schülerzahl deutlich stärker zurück als die Zahl der Schulen, was darauf hinweist, dass in der Schulstandortplanung zu dieser Zeit die Sicherung bestehender Standorte im Vordergrund steht. Man könnte dies als eine Art "passive“ Dezentralisierung bezeichnen, die dadurch entsteht, dass trotz sinkender Schülerzahlen an kleinen Standorten festgehalten wird. Dieser Erhalt der Schulen vor Ort geht mit einer Verkleinerung der Schulen einher, denn in den Kreisen, die ganz dem ländlichen Raum zuzuordnen sind, steigt der Anteil der Grundschulen, die als sog. „Kleinschulen“ mit weni- 
ger als 64 Schüler*innen geführt werden, von 24 Prozent (1991/92) auf 33 Prozent $(2014 / 15)$. Sie stellen damit die vorherrschende Größenkategorie der Schulen in diesen Kreisen dar. Somit reagiert das Schulstandortnetz bis 2014/15 auf die sinkenden Schülerzahlen vorwiegend mit einer Verkleinerung der Schulen und jahrgangsübergreifendem Unterricht. Im Schuljahr 2014/15 liegt der Klassenteiler für Grundschulen bei 28 Schüler*innen und die Mindestschülerzahl für eine Grundschulklasse bei 16 Schüler*innen, welcher mit der Einrichtung jahrgangsübergreifender Klassen an peripheren Standorten Rechnung getragen wird. Richtet man den Blick auf die Gemeinden, so ist ein Ergebnis dieser Maßnahmen, dass im Jahr 2014/15 nur in 63 Gemeinden (und damit sechs Prozent aller Gemeinden des Landes) keine Grundschule vor Ort besteht, wovon 58 Gemeinden dem ländlichen Raum zuzurechnen sind. Sie liegen vorwiegend auf der Schwäbischen Alb oder im südlichen Hochschwarzwald, und ihre Einwohnerzahl liegt in 87 Prozent der Fälle unter 1.000 Einwohner*innen. Sie besaßen bereits 1991/92 keine eigene Schule, so dass im Untersuchungszeitraum keine weitere Gemeinde ihre letzte Grundschule verlor ganz im Gegenteil: Von den 76 Gemeinden ohne Grundschule im Jahr 1991/92 erhielten bis 2014/15 sieben Gemeinden wieder eigene Grundschulen.

Nutzt man für den Vergleich zwischen den Schuljahren 1991/92 und 2014/15 die in Abb. 1 und Abb. 2 eingeführte Typisierung der Standortentwicklung für alle 1.101 Gemeinden (Abb. 5), so werden kleinräumige Muster der Zentralisierungs- und Dezentralisierungsprozesse sichtbar. In dieser Karte ist für jede Gemeinde der Quotient aus Schüler- und Schulenzahl in seiner Entwicklung zwischen 1991/92 und 2014/15 abgebildet. Das heißt, wenn in einer Gemeinde trotz sinkender Schülerzahl die Schulenzahl ansteigt, gleich bleibt oder in gleichem Maße wie die Schülerzahl sinkt, dann zählt sie zu dem Typus „Dezentralisierung bei sinkenden Schülerzahlen (DEZsinkend)“ ( $n=575)$; steigen beide Zahlen, dann zählt sie zum Typus „Dezentralisierung bei steigenden Schülerzahlen (DEZ-steigend)“ $(n=16)$; sinken beide Zahlen, zählt sie zu „Zentralisierung bei sinkenden Schülerzahlen (ZENTR-sinkend)“ $(\mathrm{n}=79)$; sinkt die Schulenzahl, bleibt sie gleich oder steigt deutlich weniger an als die Schülerzahl, so zählt sie zu "Zentralisierung bei steigenden Schülerzahlen (ZENTRsteigend)“ $(n=95)$. Bewegen sich die Veränderungen im Bereich von $+/-10$ Prozent, so wird dies als „stabil“ bezeichnet $(n=336)$. Grundsätzlich ist zu erkennen, dass der Typus „DEZ-sinkend“ mehr als die Hälfte der Gemeinden ausmacht (52\%). Dies bedeutet, dass in den meisten Gemeinden trotz sinkender Schülerzahlen die Zahl der Schulen nur wenig oder gar nicht reduziert wurde, so dass man von einer „passiven Dezentralisierung“ sprechen kann. Diese Gemeinden befinden sich im Osten und Nordosten des Landes sowie im Schwarzwald, d.h. in ländlichen Regionen. Daran lässt sich erkennen, dass man in dem beobachteten Zeitraum in diesen Regionen den demographischen Prozessen mit strukturellen Anpassungen begegnet ist, die auf den Erhalt der Schulen Wert legten. Auf der Schwäbischen Alb, in der Region Hohenlohe und im Breisgau sind dagegen Zentralisierungstendenzen sichtbar. Hier wurden in den Gemeinden Schulen geschlossen, was sowohl mit sinkenden Schülerzahlen, häu- 
Abb. 5: Typen der Standortentwicklung öffentlicher Grundschulen in Gemeinden zwischen 1991/92 und $2014 / 15$

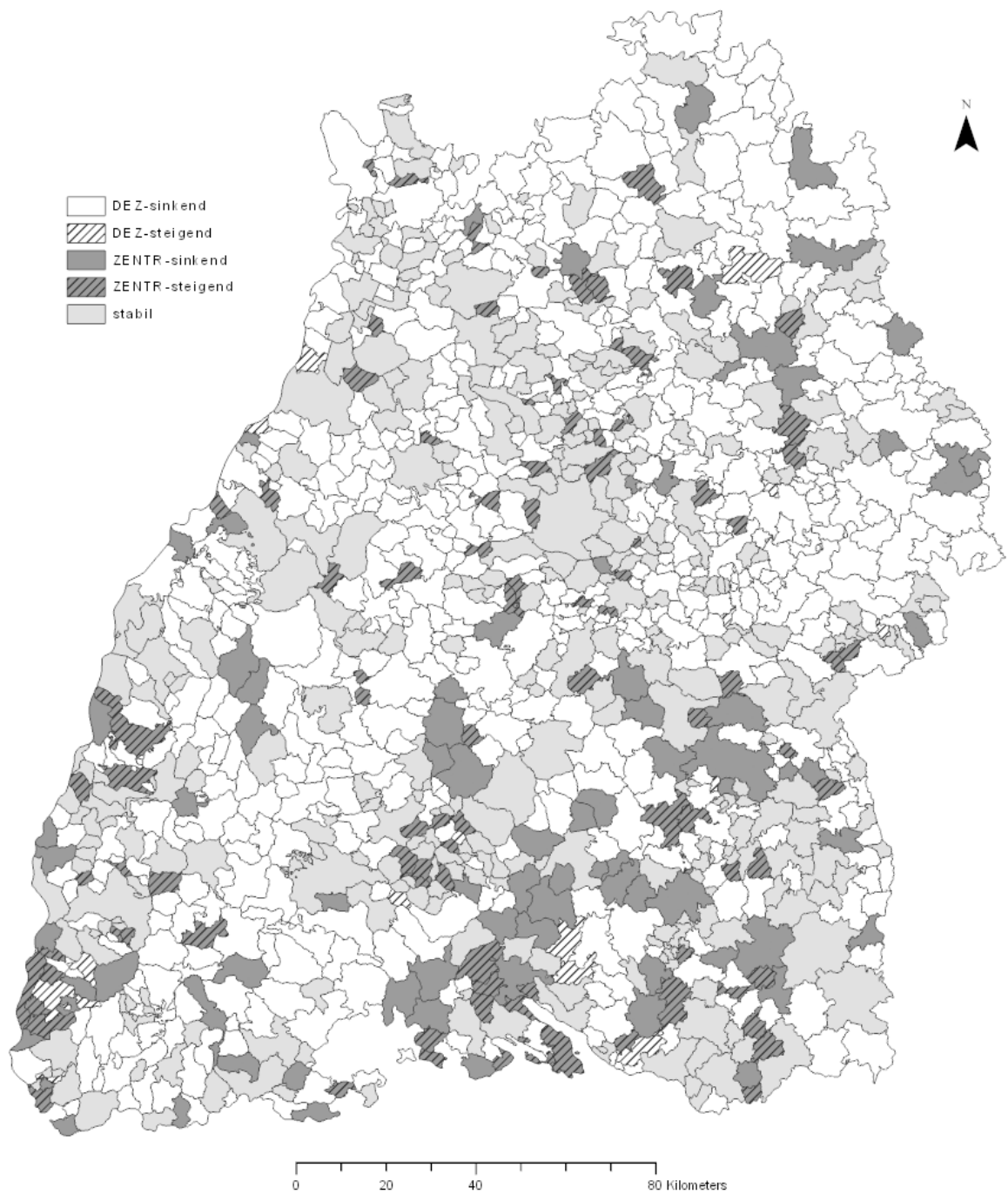

Quelle: eigener Entwurf, nach Sonderauswertungen des Statistischen Landesamtes

figer jedoch sogar mit steigenden Schülerzahlen einherging. Dies deutet darauf hin, dass dort eine andere Schulstandortpolitik betrieben wurde, die weniger den Erhalt der Ortsteilschulen im Blick hatte. Es ist zu vermuten, dass dies auf Entscheidungen in den Regierungsbezirken oder den Schulämtern zurückzuführen ist, d.h. auf bildungspolitische Leitbilder und Strategien. 


\section{Wie reagiert man in Baden-Württemberg auf die jüngsten Entwicklungen?}

In den Jahrzehnten nach der Schließungswelle der 1960-/70er-Jahre erwies sich das Standortnetz der Grundschulen in Baden-Württemberg als relativ stabil, obwohl Zuund Abnahmen der Schülerzahlen stattfanden. Man hat im Rahmen des bestehenden Schulstandortnetzes weitgehend so flexibel reagiert, dass in Wachstumsphasen bestehende Schulen vergrößert und in Schrumpfungsphasen Schulen entweder verkleinert, $\mathrm{zu}$ Außenstellen umgewandelt wurden und/oder jahrgangsübergreifender Unterricht eingeführt wurde.

In der Arbeit von Bauer (2016) wurde auch geprüft, ob die Gemeinden, in denen im Rahmen des Programms zur „Wiedereinrichtung wohnortnaher Grundschulen“ von 1987 bis 1992 Schulen (wieder-)eröffnet wurden, ihre Schulen erhalten konnten, oder ob dieser Phase der Dezentralisierung wenig später erneut eine Zentralisierung folgte. In zwei Dritteln der Gemeinden (64 von 97) blieb die Zahl der Schulen gleich, in 15 Fällen wuchs die Zahl der Schulen, und nur in 18 Gemeinden nahm die Zahl der Schulen ab (ebd., S. $111 \mathrm{f}$.). Insofern hat das damalige Programm langfristig nachhaltig zur Verbesserung der schulischen Versorgung vor allem in ländlichen Regionen beigetragen.

Allerdings gelten nach den Kriterien der Ständigen Kultusministerkonferenz der Länder aus dem Jahr 2014 (KMK, 2014, S. 2), die sich an Mindestschülerzahlen und Klassenbesetzungen orientieren, 22 Prozent aller Grundschulen in BadenWürttemberg und rund 30 Prozent in ländlichen Räumen, in einzelnen Kreisen sogar 40 Prozent, als gefährdet (Bauer, 2016, S. 116). Das Motto der Bildungspolitik im jüngsten Koalitionsvertrag lautet „verlässlich, vielfältig, innovativ“ (Bündnis 90/ Die Grünen BW \& CDU BW, 2016, S. 25) sowie „kurze Wege für kurze Beine“ (ebd., S. 27). Es wird sich erweisen, inwieweit die Landesregierung auch zukünftig daran festhält. Allerdings deutet der nachfolgende Text, den das Ministerium für Kultus, Jugend und Sport unter dem Stichwort „Regionale Schulentwicklung“ veröffentlicht hat, darauf hin, dass mit Schließungen kleiner Schulen zu rechnen ist:

„Baden-Württemberg steht im Hinblick auf die Weiterentwicklung seiner Bildungslandschaft weiterhin vor Herausforderungen. Eine davon ist die Anpassung der Schulstrukturen an die veränderten gesellschaftlichen Bedingungen, dabei vor allem an den demografischen Wandel und das veränderte Schulwahlverhalten.

Wichtigstes Ziel der regionalen Schulentwicklung ist es, allen Schülerinnen und Schülern in zumutbarer Erreichbarkeit von ihrem Wohnort einen Bildungsabschluss entsprechend ihren Begabungen und Fähigkeiten zu ermöglichen. Gleichzeitig geht es darum, im Interesse aller Beteiligten langfristig leistungsstarke und effiziente Schulstandorte zu sichern, gerade auch in ländlichen Gebieten. 
Es sollen Schulen geschaffen werden, die aufgrund ihrer Größe sehr gute pädagogische Bedingungen bieten und langfristig effizient arbeiten können. So können große Schulen unter anderem vielfältigere pädagogische Angebote schaffen, etwa in Hinblick auf Wahlmöglichkeiten, als kleine Schulen. Auch können Personalengpässe, etwa bei Erkrankung einer Lehrkraft, an großen Schulen besser ausgeglichen werden." (MKJS BW, 2018)

Die mehrfachen Hinweise auf die „Effizienz der Schule“ (d.h. ökonomische Effizienz bzgl. des Einsatzes der Landesmittel) und der unterstellte Zusammenhang zwischen der Qualität einer Schule und ihrer Größe entsprechen der Rhetorik, die bereits in der Landschuldiskussion des vergangenen Jahrhunderts von Verfechter ${ }^{\star}$ innen der großen Schulen verwendet wurde. Es ist bedauerlich, dass die wissenschaftlichen Erkenntnisse zur Qualität kleiner Schulen und ihrer Bedeutung für die Gemeinden hier offensichtlich keinen Eingang finden. Ebenso vermisst man in den Förder- oder Entwicklungsprogrammen für den ländlichen Raum die Erwähnung der Grundschule als zentralem Element der Infrastruktur (MLR BW, 2014), obwohl deren Existenz wesentlich über die Attraktivität als Wohnstandort für junge Familien entscheidet. Das wichtige Thema „Schulwege“ wird in dieser Darstellung mit dem Stichwort „in zumutbarer Erreichbarkeit“ nur am Rande erwähnt. Die Frage, wie weit ein zumutbarer Schulweg mit welchem Verkehrsmittel für ein Grundschulkind sein darf, wird meist nicht in einer Wegelänge oder Wegezeit bemessen, sondern von den konkreten Schulwegbedingungen abhängig gemacht. Dabei sollte berücksichtigt werden, dass ein Schulweg auch immer ein "Erlebnis-, Erfahrungs- und Lernweg“ (Limbourg, 2009, S. 26) ist, auf dem Kinder wichtige soziale und motorische Fähigkeiten erwerben. Zudem kann das Auspendeln von Kindern aus dem Wohnort die Entstehung einer lokalen Identität behindern. Langfristig fördert man damit Abwanderungstendenzen aus den kleinen Gemeinden (Kramer, 1993).

Dass manche Standorte in Zeiten des demographischen Wandels dennoch aufgrund des außerordentlichen Engagements von Bürgermeister*innen, Schulleiter ${ }^{\star}$ innen oder Eltern trotz der Konzentration des Schulstandortnetzes bestehen bleiben, lässt sich immer wieder mit Beispielen belegen. Im Detail wird oft sichtbar, dass bei einer Entscheidung für/gegen einen Schulstandort viele Faktoren und Akteure eine Rolle spielen, die berücksichtigt werden müssen. Eine Entscheidung ausschließlich nach ökonomischer Effizienz greift bei einer solch folgenreichen Veränderung für einen Ort sicher zu kurz.

Während sich das Schulstandortnetz der öffentlichen Schulen nach den Schließungen der 1960er-/70er-Jahre insgesamt als relativ stabil erwiesen hat, ist vor allem seit Mitte der 1990er-Jahre das Netz der privaten Schulen ${ }^{7}$ stark expandiert (von 1991/92 bis 2014/15 um knapp 500\%; Bauer, 2016, S. 91). Die privaten Grundschulen befin-

7 Dazu zählen nicht die Freien Waldorfschulen. 
den sich zu 70 Prozent in Verdichtungsräumen und ihren Randzonen, allen voran in Freiburg, Stuttgart, Heidelberg und Tübingen, d.h. besonders in Universitätsstädten. Interessant ist die Größe und demzufolge die Zügigkeit der privaten Grundschulen: Mehr als die Hälfte der privaten Grundschulen (53\%) sind Kleinschulen mit weniger als 64 Schüler*innen, wohingegen nur 22 Prozent aller Grundschulen in öffentlicher Trägerschaft Kleinschulen sind. Die geringe Größe der privaten Grundschulen scheint in diesem Fall offensichtlich die Attraktivität der Schulen eher zu erhöhen. In diesem Zusammenhang gilt es, auf die Ergebnisse von Kann (2016) hinzuweisen. Sie stellt fest, dass Schließungen von öffentlichen Schulstandorten (aufgrund der strengen Umsetzung von Regelungen zur Mindestschülerzahl) oft zeitgleich mit Eröffnungen neuer Privatschulen einhergehen (ebd., S. 257). Der von ihr beobachtete Prozess einer zunehmenden Segregation, der wiederum die Integrationsfunktion der Grundschule erodiert (ebd., S. 252), stimmt nachdenklich.

\section{Zusammenfassung und Desiderata}

Nach den großen Zentralisierungswellen in den 1960er-/70er-Jahren ist die absolute Zahl von Schulen im Land seit geraumer Zeit fast gleich geblieben. Dennoch deuten Verschiebungen in der Organisationsstruktur (Rückstufung von Schulen zu Außenstellen, Schließung der Sekundarstufen) sowie die Ausbreitung von Privatschulen auch im Primarbereich darauf hin, dass sich spürbare Veränderungen abzeichnen. Die Einflussfaktoren auf diese Prozesse sind vielfältig und reichen weit über den Faktor „Entwicklung der Schülerzahlen“ hinaus. Es sind Phasen erkennbar, in denen bildungspolitische Leitbilder, die durchaus ideologische Züge tragen, zum Zuge kommen (z. B. ein Modernisierungsideal während der großen Schließungswellen). In anderen Phasen werden pädagogische Konzepte bedeutsam (wie z. B. in der Phase der Wiedereröffnung der kleinen Schulen mit jahrgangsübergreifendem Unterricht), oder es werden verwaltungstechnische bzw. ökonomische Gründe als maßgeblich angesehen (evtl. auch in näherer Zukunft), mit denen Schulschließungen begründet werden. Auch ein Lehrkräftemangel oder unbesetzte Schulleitungsstellen werden immer wieder zum Anlass genommen, kleine Schulen zu schließen.

Von zentraler Bedeutung für ein dichtes Schulstandortnetz sind all die Maßnahmen, die von Seiten der Schulorganisation getroffen werden, wie z.B. niedrige und flexible Mindestschülerzahlen für Klassen und Schulen, die Einrichtung jahrgangsübergreifender Klassen, Fachlehrereinsatz an mehreren Schulen (wie z.B. in Bayern) und eine angemessene Besoldung sowie eine Deputatsreduktion für Schulleitungen in kleinen Schulen. In Nachbarländern, wie z.B. im österreichischen Bundesland Vorarlberg, werden Grundschulen (dort „Volksschulen“ genannt) auch mit weniger 
als 20 Schülern geführt. ${ }^{8}$ Dort werden Vorgaben bzgl. Mindestschülerzahlen flexibler gehandhabt, und die örtliche Volksschule genießt eine sehr hohe Wertschätzung. Lehrkräfte für kleine Schulen werden meist direkt von den Schulen angeworben und nicht von zentraler Stelle zugewiesen, was die Fluktuation deutlich reduziert. Diese Vorgehensweise kann durchaus als Vorbild für ländliche Regionen in Deutschland angesehen werden.

Nicht zu unterschätzen sind die lokalen Einflussfaktoren, die darauf hinweisen, welch eine identitätsstiftende Kraft eine Schule für eine Gemeinde besitzt, was vor allem bei drohenden Schließungen sichtbar wird. Insofern sind Akteure auf allen räumlichen Ebenen bei eine Analyse der Einflussfaktoren auf das Schulstandortnetz zu berücksichtigen.

Es wäre für wissenschaftliche Zwecke außerordentlich hilfreich, wenn zumindest ein Teil jener Daten, die an Schulen mit großem Aufwand zu Verwaltungszwecken erhoben werden, für die Forschung zugänglich wäre. Dazu würden z. B. Informationen der weiterführenden Schulen zählen, wie Angaben zur Herkunftsschule der Schüler*innen und zum Schulerfolg der Kinder. So ließe sich z. B. klären, ob Kinder aus kleinen Schulen mit jahrgangsübergreifendem Unterricht an weiterführenden Schulen weniger erfolgreich sind (was Kleinschulgegner*innen unterstellen) oder ob sie gleiche oder sogar bessere Ergebnisse erzielen als Kinder aus großen Schulen (was in Expertengesprächen mit Lehrkräften aus weiterführenden Schulen mehrfach angedeutet wurde).

Grundsätzlich deutet vieles darauf hin, dass eine auf ökonomische Effizienz ausgerichtete Bildungsplanung große Risiken birgt. Grundschulen am Wohnort der Kinder erfüllen wichtige Funktionen, die sowohl aus der Perspektive der Kinder als auch aus der Perspektive der Schulstandorte prägend sind. Aus diesem Grund muss sich die Bildungs- und damit auch die Schulstandortplanung die Frage stellen lassen, welche Ziele eine Gesellschaft mit Hilfe dieser Institution verfolgt und in wessen Hand sie ihre Gestaltung legt.

\section{Literatur und Internetquellen}

Åberg-Bengtsson, L. (2009). The Smaller the Better? A Review of Research on Small Schools in Sweden. International Journal of Educational Research, 48, 100-108. https://doi. org/10.1016/j.ijer.2009.02.007

8 Im Schuljahr 2016/17 wurden von den 168 Vorarlberger Volksschulen 101 Schulen $(=60 \%)$ mit 14.379 Schüler ${ }^{*}$ innen $(=84 \%)$ vollorganisiert in Jahrgangsklassen geführt. Jahrgangsübergreifender Unterricht fand in 67 Schulen $(=40 \%)$ für 2.629 der Schüler*innen $(=16 \%)$ statt. In die Kategorie Kleinstschulen mit 20 und weniger Schüler*innen fielen 21 Schulen (darunter zwei Privatschulen), und in weiteren 45 Schulen lag die Schülerzahl unter 40 Schüler*innen (Amt der Vorarlberger Landesregierung, 2018). 
Amt der Vorarlberger Landesregierung (Hrsg.). (2018). Schulstatistik 2016/2017. Bregenz.

Autti, O., \& Hyry-Beihammer, E. K. (2014). School Closures in Rural Finnish Communities. Journal of Research in Rural Education, 29 (1), 1-17.

Bauer, L. (2016). Die Entwicklung des Standortnetzes von Grundschulen in Baden-Württemberg von 1991-2014. Unveröffentlichte Zulassungsarbeit zum 1. Staatsexamen. Karlsruhe: KIT, Institut für Geographie und Geoökologie.

Bündnis 90/Die Grünen BW \& CDU BW (2016). Baden-Württemberg gestalten: Verlässlich, Nachhaltig, Innovativ. Koalitionsvertrag zwischen Bündnis 90/Die Grünen BadenWürttemberg, CDU Baden-Württemberg 2016-2021. Stuttgart.

Bundesministerium für Justiz und Verbraucherschutz \& Bundesamt für Justiz (2008). Raumordnungsgesetz. Zugriff am 13.4.2018. Verfügbar unter: https://www.gesetze-iminternet.de/rog_2008/__.html.

Diederich, J. (1967). Die Landschulreform im Spiegel der pädagogischen Zeitschriften. Weinheim: Beltz.

Fickermann, D. (1997). Soziale Aspekte der Bildungsbeteiligung. In P. Zedler \& H. Weishaupt (Hrsg.), Kontinuität und Wandel. Thüringer Schulen im Urteil von Schülern, Lehrern und Eltern (S. 147-170). Weinheim: Beltz.

Fickermann, D., Schulzeck, U., \& Weishaupt, H. (2002). Unterschiede im Schulbesuch. In A. Mayr \& M. Nutz (Hrsg.), Bildung und Kultur. Nationalatlas Bundesrepublik Deutschland, Bd. 6 (S. 40-43). Heidelberg \& Berlin: Spektrum.

Fickermann, D., Weishaupt, H., \& Zedler, P. (Hrsg.). (1998). Kleine Grundschulen in Europa. Berichte aus elf europäischen Ländern. Weinheim: Deutscher Studien Verlag.

Freytag, T., Jahnke, H., \& Kramer, C. (2015). Bildungsgeographie. GeoWissen Kompakt. Darmstadt: Wissenschaftliche Buchgesellschaft.

Freytag, T., Jahnke, H., \& Kramer, C. (2016). Geographische Bildungsforschung. In D. Fickermann \& H.-W. Fuchs (Hrsg.), Bildungsforschung - disziplinäre Zugänge: Fragestellungen, Methoden und Ergebnisse (S. 139-155). Münster et al.: Waxmann.

Jahnke, H., Kramer, C., \& Meusburger, P. (Hrsg.). (2019, im Druck). Geographies of Schooling (Knowledge and Space 14). Heidelberg: Springer.

Kann, C. (2016). Schulschließungen und der Umbau von Schulstandorten. Steuerungsansätze bei sinkenden Schülerzahlen und die Rolle der Privatschulen. Wiesbaden: Springer VS. https://doi.org/10.1007/978-3-658-18269-4_10

KMK (Sekretariat der Ständigen Konferenz der Kultusminister der Länder in der Bundesrepublik Deutschland) (2014). Vorgaben für die Klassenbildung. Schuljahr 2014/2015. Berlin: KMK.

Kramer, C. (1993). Die Entwicklung des Standortnetzes von Grundschulen im ländlichen Raum. Vorarlberg und Baden-Württemberg im Vergleich (Heidelberger Geographische Arbeiten 93). Heidelberg: Geographisches Institut der Universität Heidelberg.

Kvalsund, R., \& Hargreaves, L. (2009). Reviews of Research on Rural Schools and Their Communities: Analytical Perspectives and a New Agenda. International Journal of Educational Research, 48, 140-149. https://doi.org/10.1007/978-3-662-39463-2_3

Landtag Baden-Württemberg, Drucksache 16/758 (2016). Antrag der Abg. Gerhard Kleinböck u. a., SPD, und Stellungnahme des Ministeriums für Kultus, Jugend und Sport. Entwicklung der baden-württembergischen Werkreal- und Hauptschulen und Perspektiven für ihre Lehrkräfte. Zugriff am 22.04.2018. Verfügbar unter: https://www.land tag-bw.de/files/live/sites/LTBW/files/dokumente/.../16_0758_D.pdf.

Lehrer Online in Baden-Württemberg. Zugriff am 16.04.2018. Verfügbar unter: https://www. lehrer-online-bw.de/,Len/Startseite/module/sls.

Limbourg, M. (2009). Lernort Schulweg. Die Grundschulzeitschrift, 224, 26-31.

Meusburger, P. (1998). Bildungsgeographie. Wissen und Ausbildung in räumlicher Dimension. Heidelberg \& Berlin: Spektrum. 
MKJS BW (Ministerium für Kultus, Jugend und Sport Baden-Württemberg) (2018). Regionale Schulentwicklung. Zugriff am 26.04.2018. Verfügbar unter: http://www.kmbw.de/rse.

MKJS BW (Ministerium für Kultus, Jugend und Sport Baden-Württemberg) (o. D.). Ganztagsschule in Baden-Württemberg. Zugriff am 18.02.2019. Verfügbar unter: http:// ganztagsschule-bw.de/,Lde/Startseite.

MLR BW (Ministerium für ländlichen Raum und Verbraucherschutz Baden-Württemberg) (Hrsg.). (2014). Entwicklungsprogramm Ländlicher Raum (ELR). Zugriff am 22.04.2018. Verfügbar unter: http://www.lel-bw.de/pb/MLR.Foerderung,Lde/Startseite/Foerder wegweiser/Entwicklungsprogramm+Laendlicher+Raum+_ELR_.

Picht, G. (1964). Die deutsche Bildungskatastrophe. Analyse und Dokumentation. Freiburg i. Br.: Walter.

Raggl, A. (2015). Teaching and Learning Practices in Small Rural Schools in Austria and Switzerland - Opportunities and Challenges from Teachers' and Students' Perspectives. International Journal of Educational Research, 74, 127-135. https://doi.org/10.1016/j. ijer. 2015.09.007

Raggl, A., Smit, R., \& Kerle, U. (Hrsg.). (2015). Kleine Schulen im ländlich-alpinen Raum (FokusBildungSchule, Bd. 8). Innsbruck: Studien Verlag.

Sixt, M. (2010). Regionale Strukturen als herkunftsspezifische Determinanten von Bildungsentscheidungen. Kassel: Universität Kassel. Zugriff am 26.04.2018. Verfügbar unter: https://kobra.bibliothek.uni-kassel.de/handle/urn:nbn:de:hebis:34-2010110934909.

Statistisches Landesamt Baden-Württemberg (Hrsg.). (Diverse Jahrgänge). Berichte/B/I/1, Allgemeinbildende Schulen in Baden-Württemberg im Schuljahr ... Unterricht und Bildung. Stuttgart. Zugriff am 16.04.2018. Verfügbar unter: https://www.destatis.de/ GPStatistik/receive/BWSerie_serie_0000058.

Weichhart, P., Weiske, C., \& Werlen, B. (2006). Place Identity und Images. Das Beispiel Eisenhüttenstadt (Abhandlungen zur Geographie und Regionalforschung 9). Wien: Institut für Geographie und Regionalforschung.

Werlen, B. (2000). Sozialgeographie. Eine Einführung. Bern: Haupt.

Wirtschaftsministerium Baden-Württemberg (Hrsg.). (2002). Landesentwicklungsplan 2002. Stuttgart: Ministerium.

Caroline Kramer, Prof. Dr., geb. 1961, Univ.-Professorin für Humangeographie, Institut für Geographie und Geoökologie am Karlsruher Institut für Technologie (KIT).

E-Mail: caroline.kramer@kit.edu

Anschrift: KIT, Kaiserstr. 12, 76131 Karlsruhe

Linda Bauer, geb. 1992, Studienreferendarin am Burghardt-Gymnasium Buchen.

E-Mail: linda.bauer@bgbuchen.de

Anschrift: Burghardt-Gymnasium, St.-Rochus-Straße 5, 74722 Buchen 